\title{
Magnetic wires: A novel design for neuroendovascular coil retrieval
}

\author{
LINGYUN ZHANG and YUGANG JIANG
}

Neurosurgery Department, The Second Xiangya Hospital of Central South University, Changsha, Hunan 410011, P.R. China

Received October 17, 2016; Accepted April 11, 2017

DOI: $10.3892 /$ etm.2018.6503

\begin{abstract}
The present study aimed to evaluate the operation, application and safety of a novel prototype device designed for the retrieval of a migrated coils, an intraprocedural complication of endovascular aneurysm treatment, compared with the widely used Solitaire AB stent retriever. The prototype retrieval device, referred to as magnetic wires, consisted of two microwires that were joined by a small magnetic ring near to the tips. To retrieve a coil, the wires are advanced through the dislodged coil one at a time and then capture the target coil in a chopstick-like manner, as their tips are attracted to the magnet. In vitro tests were performed five times for each retrieval device using a pulsing flow circulator to compare retrieval effectiveness. In vivo efficacy testing was performed in 5 male piglets. The left and right carotid arteries were used to assess the magnetic wires and the Solitaire retriever, respectively. While the two devices successfully retrieved coils in vitro at a rate of $100 \%$, the magnetic wires did so in a shorter time. In vivo, the magnetic wires successfully captured the migrated coils in $3 / 5$ cases $(60 \%)$, whereas the Solitaire AB stent retriever was successful 4/5 cases $(80 \%)$. Endothelial injury was observed following magnetic wires retrieval in $1 / 5$ cases $(20 \%)$ and following use of the Solitaire AB stent retriever in $3 / 5$ cases $(60 \%)$. These results demonstrate the feasibility and safety of this novel prototype coil retrieval device, which with further development has the potential to be an optimal device for the retrieval of dislodged coils.
\end{abstract}

Correspondence to: Professor Yugang Jiang, Neurosurgery Department, The Second Xiangya Hospital of Central South University, 139 Renmin Road, Changsha, Hunan 410011, P.R. China E-mail: ybblj@hotmail.com

Abbreviations: LM, light microscopy; SEM, scanning electron microscopy; SmCo, samarium cobalt

Key words: neuroendovascular, aneurysm, complications, dislodged coil, retrieval device

\section{Introduction}

Cerebral aneurysms are present in an estimated $6 \%$ of the global population $(1,2)$. Cerebral aneurysms rupture in $1 \%$ of these patients, which, depending on their size, ultimately lead to subarachnoid hemorrhage (3). The 1-year mortality rate was $75 \%$ in 6,850 patients with saccular, but untreated ruptured intracranial aneurysms admitted to hospital for a week between 1968 and 2007 in Finland (4). Traditional clipping and emergence coiling are the two necessary treatment measures applied to eliminate cerebral aneurysms (1). The results of the International Subarachnoid Aneurysm Trial (ISAT) $(5,6)$ and the International Study of Unruptured Intracranial Aneurysms (ISUIA) (1) indicated that overall morbidity and mortality of patients after a 1-year follow-up that received endovascular coiling was lower than those that received surgical clipping. The associated complications of coiling have become more common, as it has become a more acceptable method that is used for the majority of patients.

Coil migration is a lethal intraprocedural complication of endovascular aneurysm treatment; however, there is no standard method for managing coil migration (7). Although the majority of physicians administer anticoagulants to address coil migration, certain physicians will use a stent to localize a dislodged coil into a large vessel (8), while others will retrieve coils via surgical or endovascular methods (9). Hopf-Jensen et al (10) demonstrated that the Solitaire ${ }^{\mathrm{TM}} \mathrm{AB}$ Neurovascular Remodeling Device exhibited a number of advantages over other devices for the retrieval of migrated coils, including large cell retriever properties and electrolyte detachment design. These results are similar to those from Liu et al (11), who analyzed Trevo Stentriever, which is another frequently used stent. In addition to the inherent stiffness and inconvenience of migrated stents, vessel damage can be caused by stent movement (12).

The present study aimed to develop a novel device for resolving coil migration that was simple to assemble, easy and safe to use, and presented a lower risk to fragile arteries in the brain. The current study designed a novel device, termed magnetic wires, consisting of two microwires, the tips of which were attached with small magnetic rings. A prototype device was constructed from commercially available materials. The feasibility and safety of this device compared with the Solitaire ${ }^{\mathrm{TM}} \mathrm{AB}$ stent retriever was evaluated in vitro and in vivo. 


\section{Materials and methods}

Prototype production and operation. A Mirage ${ }^{\mathrm{TM}} .008^{\prime}$ Hydrophilic Guidewire (Micro Therapeutics, Inc., Irvine, CA, USA) was attached to a small ( $0.8 \mathrm{~mm}$ outside diameter; $0.25 \mathrm{~mm}$ inside diameter; $1 \mathrm{~mm}$ thickness) samarium cobalt magnetic ring (SmCo; Wanxuan Magnet Company, Shenzheng, China) using ethyl- $\alpha$-cyanoacrylate glue (Hubei Huitian New Materials Co., Ltd., Shanghai, China). The microwires of the device may be advanced through the dislodged coil one at a time to retrieve the coil via the attraction of the magnetic tips, by forming a loop similar to the configuration of chopsticks (Fig. 1).

In vitro assessment. A Portable FlowTek A-215 Pulsating Flow Circulator (DialAct Corporation, Fremont, CA, USA) was used to compare the prototype with the Solitaire ${ }^{\mathrm{TM}} \mathrm{AB}$ stent retriever (Medtronic, Minneapolis, MN, USA). The pulsatile pump pushes saline at a rate of $50 \mathrm{~cm} / \mathrm{sec}$ to simulate arterial blood flow. The insect Axium ${ }^{\mathrm{TM}}$ Detachable Coil $(4 / 8 \mathrm{~mm}$; Medtronic) was inserted into the tube of the circulator through a one-way valve. The corresponding retrieval device was then pushed through the valve to catch the coil. The stent microtube was then Sent through, exceeding the coil and and the tube was then withdrawn to open the stent (like a net) to catch and retrieve the coil. The coil was then detached and withdrawn by pulling out the push-pole. The method, which uses magnetic wires to retrieve the coil, is described in Fig. 1. For A total of five retrieval trials were conducted for each device. The time from the release to the return of the coil to the tube entrance was recorded.

Animal preparation. All procedures were conducted according to the National Institutes of Health (Bethesda, MA, USA) guidelines (13) for the humane handling of animals and were approved by the Animal Care and Use Committee of The Second Xiangya Hospital of Central South University (Changsha, China). A total of 5 male healthy piglets (age, 2 months; weight, 15-17 kg) were obtained from the Experimental Pig Production Institute (Laboratory Animal Center of PLA General Hospital, Beijing, China). Pigs were housed at a temperature of $18-25^{\circ} \mathrm{C}$ and a humidity of $40-60 \%$, with a $12 \mathrm{~h} \mathrm{light/dark} \mathrm{cycle.} \mathrm{Food} \mathrm{was} \mathrm{provided} \mathrm{twice} \mathrm{a} \mathrm{day} \mathrm{at}$ $2-3 \%$ of the pigs' total body weight, with free access to water. General anesthesia was induced using pentobarbital sodium (Merck KGaA, Darmstadt, Germany) $(25 \mathrm{mg} / \mathrm{kg}$, intravenously) in $100 \%$ oxygen and a 6-F femoral artery sheath was inserted into each animal's right femoral artery following successful Seldinger puncture (14).

Coil release and retrieval. An angiogram was performed after a 6-F guiding catheter (Envoy ${ }^{\circledR}$ Guiding Catheter; Cordis Neurovascular, Inc., Miami, FL, USA) was placed in the proximal carotid artery. The DSA machine can estimate the diameter of vessel, using a metal ball, which may be more accurate. Therefore, the diameter of the carotid artery was measured via angiography using a $1 \mathrm{~cm}$ diameter iron ball (Shandong Donge Steel Ball Group Co., Ltd., Shangdong, China) attached to the right side of the head as a reference. Following the insertion of an Axium ${ }^{\mathrm{TM}}$ Detachable Coil into the carotid artery, the retrieval procedure was initiated. The coil, which was released into the left and right carotid arteries, was carefully withdrawn using the magnetic wires or the Solitaire ${ }^{\mathrm{TM}} \mathrm{AB}$ stent retriever as aforementioned (Figs. 2 and 3). The time from coil release to retrieval was recorded for each retrieval system.

All in vivo assessments were performed under X-Ray guidance. Using a digital subtraction angiography machine (Innova IGS 530; GE Healthcare, Chicago, IL, USA), the spring coil could be observed. Furthermore, the magnetic micro-conductance wires could be manipulated to pass through the gap of the spring ring under $\mathrm{X}$-ray fluoroscopy, making the magnet end of the micro guidewire connect closely.

Histopathological evaluation of coil retrieval method safety. Following sacrifice of all animals with $20 \mathrm{ml}$ potassium chloride (Jinzhong Development Zone China capital Chemical Technology Co., Ltd., Shaanxi, China; 100 g/l) intravenously, specimens were collected via neck dissection and craniotomy. From the middle portion of the carotid artery, where the retrieval was conducted, two specimens were obtained from each animal. Of these specimens, those for light microscopy (LM) were fixed using $4 \%$ formaldehyde solution for $48 \mathrm{~h}$ at $22^{\circ} \mathrm{C}$. The samples were embedded in paraffin and $4 \mu \mathrm{m}$ sections were sliced. Standard histological methods (washing with xylene) were used in order to remove paraffin and the samples were rehydrates with a graduated alcohol series. Masson's trichrome staining was performed using a Masson's trichrome kit (cat. no. ab150686; Abcam, Cambridge, UK) according to the manufacturer's protocol to identify collagen fibers within vessels. Specimens for scanning electron microscopy (SEM) were immersed in $4 \%$ paraformaldehyde for $4 \mathrm{~h}$ at $22^{\circ} \mathrm{C}$, fixed using $1 \%$ glutaraldehyde for $2 \mathrm{~h}$ at $22^{\circ} \mathrm{C}$. They were then dehydrated in a series of graded alcohol, coated with platinum-palladium using an ion coater (cat. no. IB-5; Eiko Engineering Co., Ltd., Hitachinaka, Japan). The tissues were observed using a scanning electron microscope (cat. no. S-2380N; Hitachi, Ltd., Tokyo, Japan). Endothelial lesions presented as defects in the internal elastic lamina via LM and as denudation of the wavy endothelial surface via SEM.

\section{Results}

In vitro assessment. The magnetic wires and Solitaire ${ }^{\mathrm{TM}} \mathrm{AB}$ stent retriever were able to successfully retrieve the coils released in vitro (100\%; Table I). However, the magnetic wires had a shorter retrieval time compared with the stent retrieval system (Table I).

In vivo assessment. The magnetic wires were successful in $3 / 5(60 \%)$ of the in vivo retrieval trials (Table I). However, the Solitaire ${ }^{\mathrm{TM}} \mathrm{AB}$ stent retriever successfully captured the migrated coil in $4 / 5(80 \%)$ of the trials (Table I). There were no incidences of vasospasm or vessel perforation in either group when comparing angiograms prior to and following surgery.

Histopathological safety. Specimens from the right and left carotid arteries, in which the Solitaire ${ }^{\mathrm{TM}} \mathrm{AB}$ stent retriever and magnetic wires were employed, respectively, were collected from each animal, and processed for LM and SEM. 

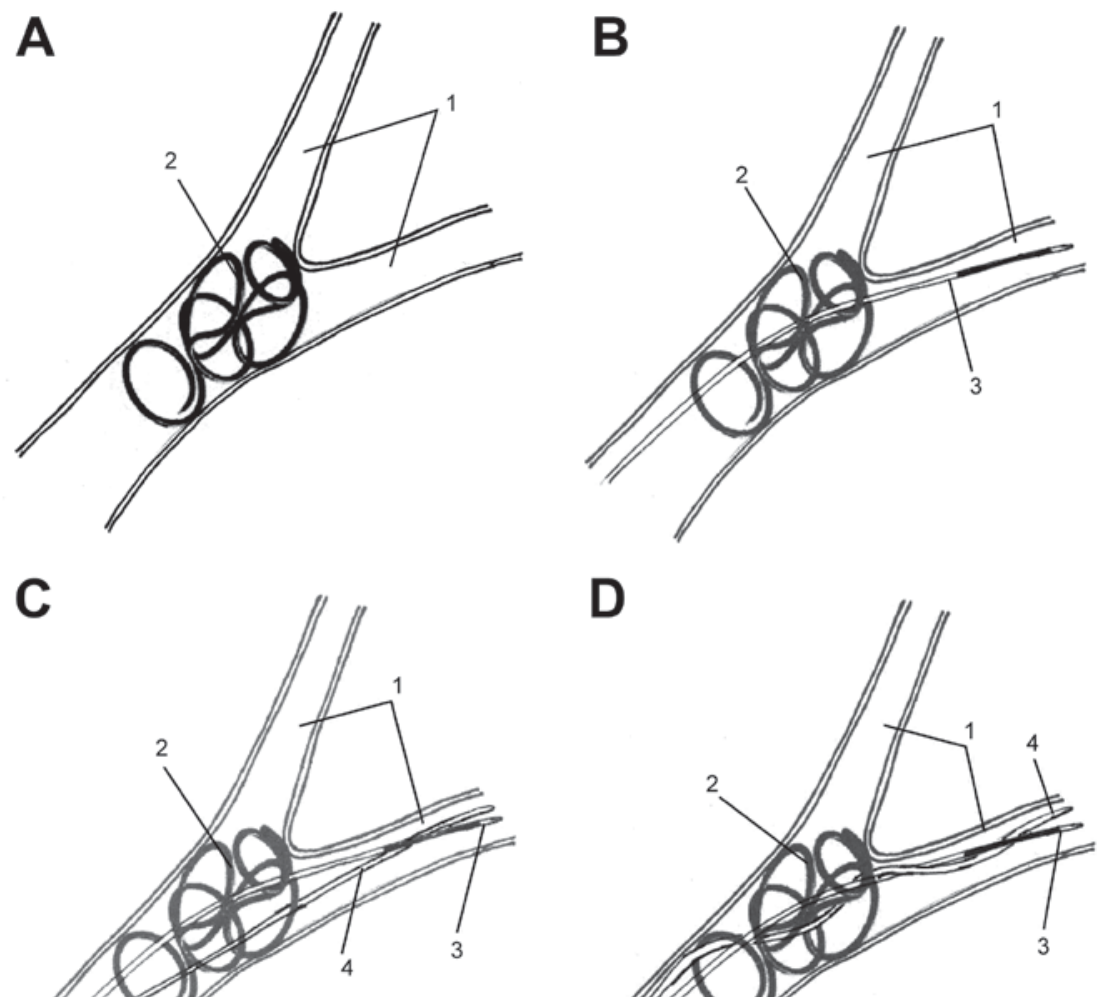

Figure 1. Application of the two-wire magnetic coil retrieval device. (A) A coil dislodged in a blood vessel. (B) Initially, one magnetic microwire is advanced through the coil. (C) The second magnetic microwire is then advanced through the coil. (D) The two wires are twisted such that the tips form a magnetically attractive loop and the coil can be retrieved. 1, Blood vessels; 2, dislodged coil; 3, first microwire; 4, second microwire.
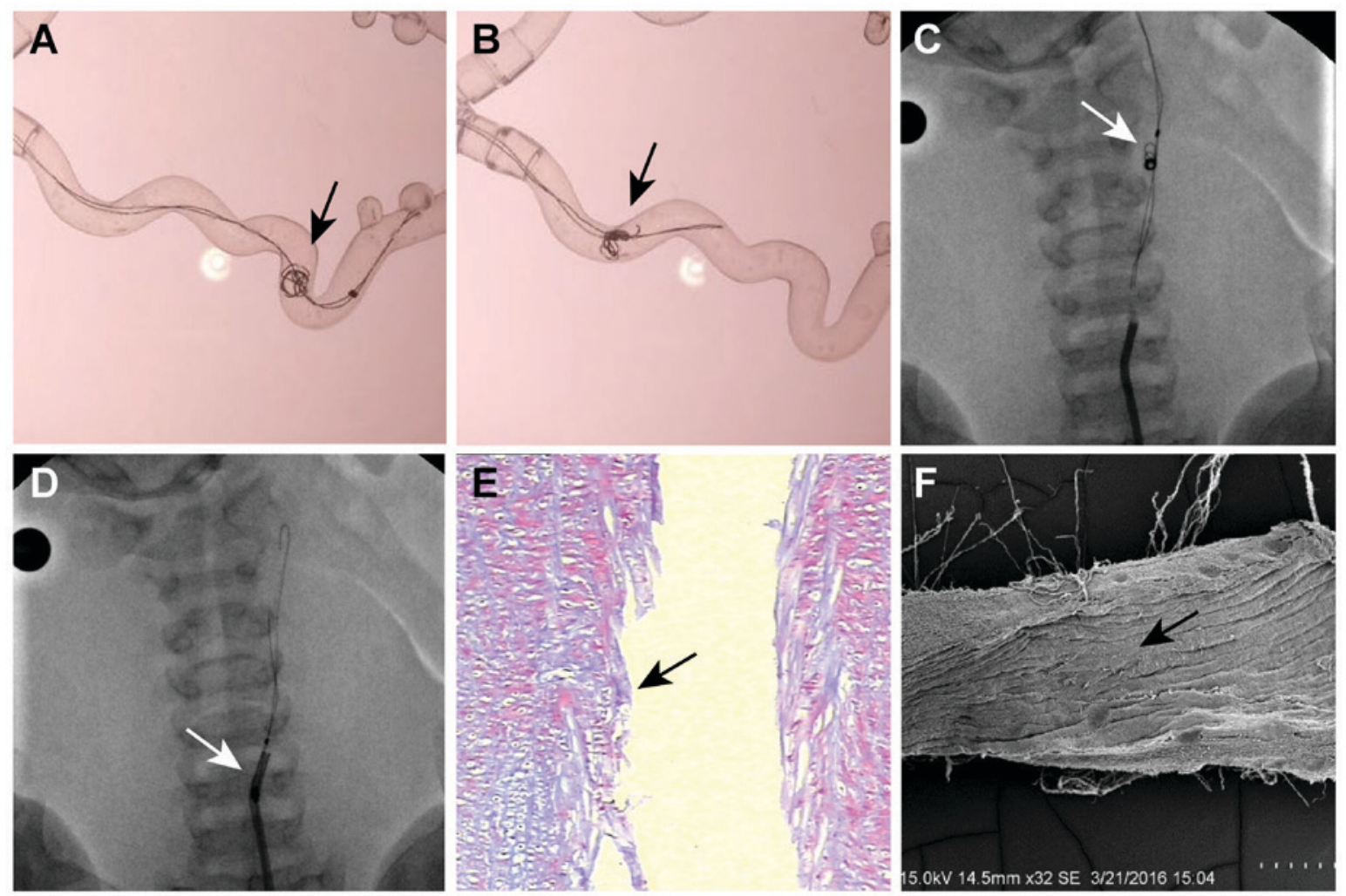

Figure 2. In vitro and in vivo assessment of the effectiveness and safety of the magnetic wires. (A) In vitro assessment, the two magnetic microwires were advanced through the migrated coil (arrow), with their tips attracted to one another by the magnetic rings to form a loop. (B) In vitro assessment, the dislodged coil was pulled back to withdraw the magnetic wires as their tips were attracted together through magnetic forces. (C) In vivo assessment, under X-Ray guidance the microwires were advanced through the migrated coil (arrow). (D) In vivo assessment, the migrated coil (arrow) was retrieved using the two magnetic microwires (X-Ray guidance). (E) Preserved intimal structures (arrow) were observed in specimens from the left carotid artery site (light microscopy; Masson's trichrome stain; magnification, x100). (F) Specimens from the left carotid artery site exhibited normal endothelial structures (arrow) without endothelial damage (scanning electron microscopy; magnification x32,000). 
Table I. Coil retrieval time using the magnetic wires and the Solitaire ${ }^{\mathrm{TM}} \mathrm{AB}$ stent retriever in vitro and in vivo.

\begin{tabular}{llccccr}
\hline & & \multicolumn{4}{c}{ Retrieval trial, sec } \\
\cline { 3 - 7 } Condition & Device used & 1 & 2 & 3 & 4 & 5 \\
\hline \multirow{2}{*}{ In vitro } & Magnetic wires & 228 & 204 & 210 & 189 & 193 \\
\multirow{3}{*}{ In vivo } & Solitaire stent & 273 & 235 & 264 & 203 & 221 \\
& Magnetic wires & Failed & 453 & 436 & Failed & 415 \\
& Solitaire stent & 427 & Failed & 392 & 418 & 387 \\
\hline
\end{tabular}
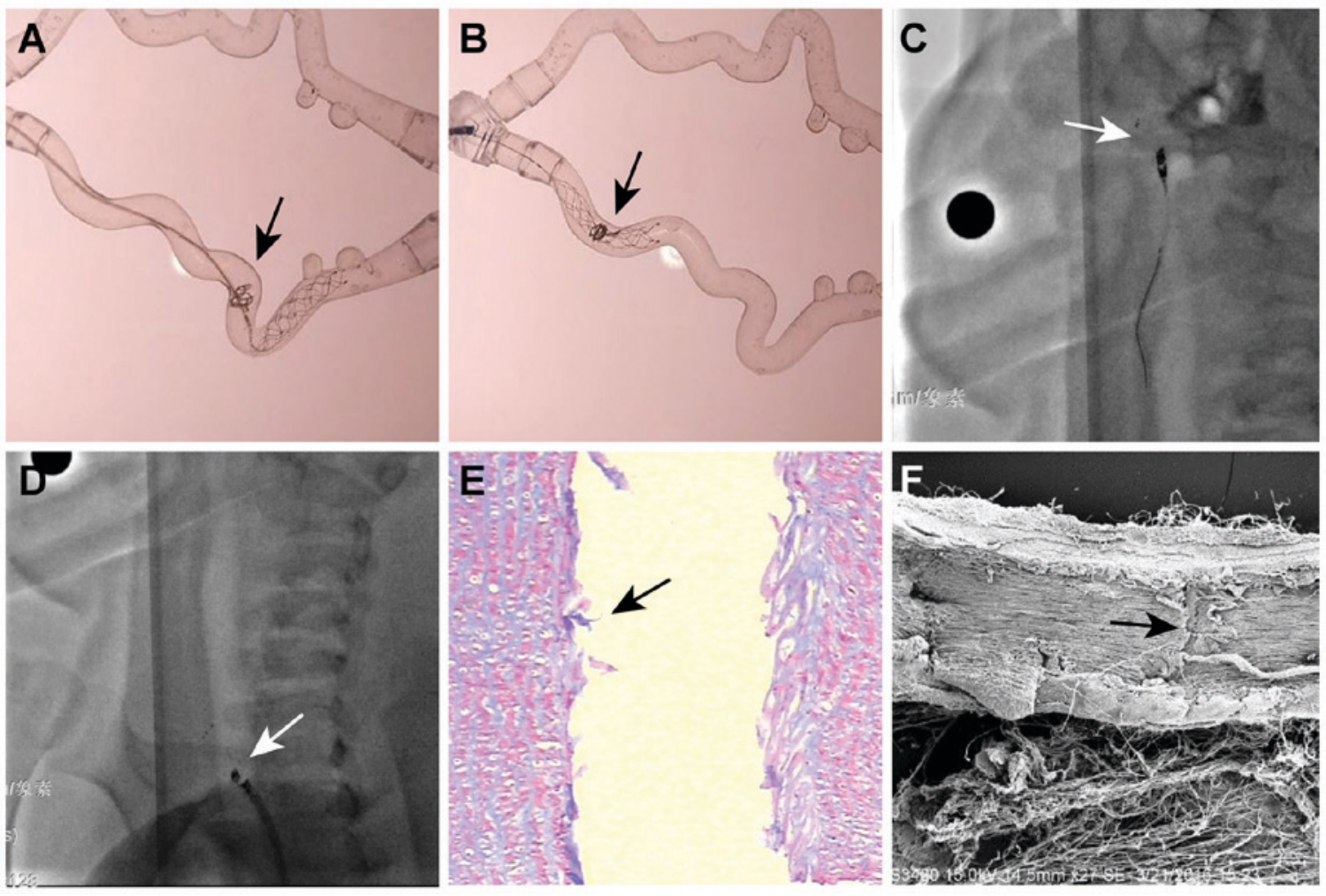

Figure 3. In vitro and in vivo assessment of the effectiveness and safety of the Solitaire ${ }^{\mathrm{TM}} \mathrm{AB}$ stent retriever. In vitro assessment demonstrated that the Solitaire ${ }^{\mathrm{TM}} \mathrm{AB}$ stent retriever (A) was advanced through the migrated coil (arrow) and (B) the dislodged coil was pulled back. In vivo assessment demonstrated that the Solitaire ${ }^{\mathrm{TM}} \mathrm{AB}$ stent retriever was also (C) advanced through and (D) retrieved the dislodged coil. (E) Endothelial lesions were detected, which presented as a defect of the endothelial internal elastic lamina (arrow) in specimens from the right carotid artery site (light microscopy; Masson's trichrome stain; magnification, x100). (F) Specimens from the right carotid artery site exhibited injury to the endothelium (arrow) (scanning electron microscopy, magnification, $\mathrm{x} 27,000)$.

Endothelial lesions were observed in $3(60 \%)$ right carotid arteries (Solitaire ${ }^{\mathrm{TM}}$ AB stent retriever) and in $1(20 \%)$ left carotid artery (magnetic wires) (Fig. 3; Table II). There was no gross intimal dissection or any abnormalities beneath the internal elastic lamina in any of the 4 cases in which endothelial lesions were observed via LM. No other specimens indicated any evidence of endothelial injury via either LM or SEM.

\section{Discussion}

There have been limited reports of the successful use of various devices for retrieving a coil that has migrated or become misplaced, including the Microsnare (15), a microcatheter retriever (Retriever-10 and Retriever-18), the Alligator
Retrieval Device $(9,15)$, Merci retriever (16), the Solitaire ${ }^{\mathrm{TM}}$ AB stent retriever (10) and the Trevo Stentriever device $(11,17)$. Several experts have shaped microwires to capture migrated coils $(18,19)$; however, the majority of clinicians use one of the following four types of coil retrieval devices: Snares, clamps, stents or spiral devices.

Existing coil retrieval devices are mechanically complex, rendering them difficult to manufacture and maneuver. Furthermore, the materials of these devices are too stiff and large for use in the fragile cerebral arteries. Watanabe et al (20) described the first use of a loop snare to retrieve a migrated coil during the treatment of a superior cerebellar artery aneurysm. However, since then, there have only been a few reported cases of snares being used to capture dislodged coils $(18,21,22)$; the lack of use of these snares may be due to difficulties in opening 
Table II. Detection of EI following the in vitro and in vivo application of the magnetic wires or the Solitaire ${ }^{\mathrm{TM}} \mathrm{AB}$ stent retriever.

\begin{tabular}{llll}
\hline \multirow{2}{*}{ Animal } & & \multicolumn{2}{c}{$\begin{array}{c}\text { Retrieval device, } \\
\text { presence of EI }\end{array}$} \\
\cline { 3 - 4 } & $\begin{array}{c}\text { Detection } \\
\text { method }\end{array}$ & $\begin{array}{c}\text { Magnetic } \\
\text { wires }\end{array}$ & $\begin{array}{c}\text { Solitaire } \\
\text { stent }\end{array}$ \\
\hline 1 & LM & No & Yes \\
2 & SEM & No & Yes \\
3 & LM & No & Yes \\
4 & SEM & No & Yes \\
4 & LM & No & No \\
5 & SEM & No & No \\
& LM & Yes & Yes \\
& SEM & Yes & Yes \\
& LM & No & No \\
\hline
\end{tabular}

EI, endothelial injury; LM, light microscopy; SEM, scanning electron microscopy.

a snare in small vessels in an appropriate position for coil retrieval. The use of clamps, including the Alligator Retrieval Device, and spiral devices, including Merci, has been reported even less frequently $(20,23)$.

The latest and the most popular type of retrieval device, the stent retrieval system, has a number of marked flaws. The recommended vessel diameter of Solitaire ${ }^{\mathrm{TM}} \mathrm{AB}$ stent retriever is from $2.2 \mathrm{~mm}$ to $6 \mathrm{~mm}$ as according to the manufacturer's protocol. Thus, it was considered that if the small artery diameter is $<2.2 \mathrm{~mm}$, the stent remains too large; and may consequently push the dislodged coil further into unreachable arterial branches. The movement of the net-like stent within the artery may damage the vessel wall, specifically endothelial cells and the internal elastic lamina, increasing the risk of inflammation and thrombosis (12). The present study also considered that when withdrawing the stent attached to the dislodged coil along the parent artery near the neck of an aneurysm, the coils in the aneurysm may be pulled out by the stent. These potential complications demonstrate that a stent-like device is not the optimal choice for coil retrieval.

The present study aimed to design an alternative type of retrieval device. The original concept of this device originated from a report describing how a number of physicians use two microwires wrapped together to capture dislodged material in a vessel (24). However, this procedure is difficult and is therefore affected by the skills of the clinician. There are a number of similarities between the two-microwire device used in the present study and chopsticks, with magnets being the key feature uniting the two microwires. The magnets used in the present study to produce the prototype device were composed of SmCo; these magnets were produced to power the stepping motor of an electronic watch and their size fits the 0.008 ' microwires.
In vitro, the prototype device demonstrated the convenience of the wire shape for advancing through the migrated coil and that the attractive force between the two magnets was sufficient for coil retrieval. These findings establish the feasibility of the magnetic wire design. However, the in vivo success rate of the novel device, in $3 / 5$ trials, was insufficient. The present study concluded that the lack of interaction with the microwires due to the enlargement of the magnetic ring at the tips resulted in difficulties controlling the device. This impedes the retrieval of a dislodged coil from an area with rapid blood flow. Furthermore, the microwire used in the current study was composed of nitinol, which contains $\sim 51 \%$ nickel and thus may be attracted to magnets; this feature of the microwire may therefore interfere with its intended function. In the present study, definite influence was observed when the second magnetic microwire tip reached and caught the former remote end. Future magnetic microwire should therefore not contain ferromagnetic material.

The LM and SEM results demonstrated that the extent of endothelial injury was smaller with the magnetic wire group compared with the Solitaire ${ }^{\mathrm{TM}}$ AB stent retriever.

In conclusion, the present study designed a simple novel device that has great potential for the retrieval of dislodged coils during interventional surgery. However, this device requires further development and validation in order to produce an optimal tool for resolving this complication of endovascular aneurysm treatment.

\section{Acknowledgements}

The present study was supported by Dr. Gary Duckwiler of the Department of Neuroradiology of David Geffen School of Medicine at UCLA (Los Angeles, CA, USA), who provided the sample coils.

\section{References}

1. Wiebers DO, Whisnant JP, Huston J III, Meissner I, Brown RD Jr, Piepgras DG, Forbes GS, Thielen K, Nichols D, O'Fallon WM, et al: Unruptured intracranial aneurysms: Natural history, clinical outcome, and risks of surgical and endovascular treatment. Lancet 362: 103-110, 2003.

2. Winn HR and Britz GW: Unruptured aneurysms. J Neurosurg 104: 179-180, 2006.

3. Broderick JP, Brott TG, Duldner JE, Tomsick T and Huster G: Volume of intracerebral hemorrhage. A powerful and easy-to-use predictor of 30-day mortality. Stroke 24: 987-993, 1993.

4. Korja M, Kivisaari R, Rezai Jahromi B and Lehto H: Natural history of ruptured but untreated intracranial aneurysms. Stroke 48: 1081-1084, 2017.

5. Molyneux A, Kerr R, Stratton I, Sandercock P, Clarke M, Shrimpton J and Holman R; International Subarachnoid Aneurysm Trial (ISAT) Collaborative Group: International subarachnoid aneurysm trial (ISAT) of neurosurgical clipping versus endovascular coiling in 2143 patients with ruptured intracranial aneurysms: A randomised trial. Lancet 360: 1267-1274, 2002.

6. Molyneux AJ, Kerr RS, Yu LM, Clarke M, Sneade M, Yarnold JA and Sandercock P; International Subarachnoid Aneurysm Trial (ISAT) Collaborative Group: International subarachnoid aneurysm trial (ISAT) of neurosurgical clipping versus endovascular coiling in 2143 patients with ruptured intracranial aneurysms: A randomised comparison of effects on survival, dependency, seizures, rebleeding, subgroups, and aneurysm occlusion. Lancet 366: 809-817, 2005.

7. Leslie-Mazwi TM, Heddier M, Nordmeyer H, Stauder M, Velasco A, Mosimann PJ and Chapot R: Stent retriever use for retrieval of displaced microcoils: A consecutive case series. AJAR Am J Neuroradiol 34: 1996-1999, 2013. 
8. Schütz A, Solymosi L, Vince GH and Bendszus M: Proximal stent fixation of fractured coils: Technical note. Neuroradiology 47: $874-878,2005$

9. Oh J, Kim J, Hong S, Hu C, Pyen J, Whang K, Cho S and You DS: Retrieval of unintended migrated detached coil: Case report. J Cerebrovasc Endovasc Neurosurg 16: 268-274, 2014

10. Hopf-Jensen S, Hensler HM, Preiss M and Muller-Hulsbeck S Solitaire ${ }^{\circledR}$ stent for endovascular coil retrieval. J Clin Neurosci 20: 884-886, 2013.

11. Liu KC, Ding D, Starke RM, Geraghty SR and Jensen ME: Intraprocedural retrieval of migrated coils during endovascular aneurysm treatment with the trevo stentriever device. J Clin Neurosci 21: 503-506, 2014.

12. Park S, Hwang SM, Song JS, Suh DC and Lee DH: Evaluation of the solitaire system in a canine arterial thromboembolic occlusion model: Is it safe for the endothelium? Interv Neuroradiol 19 417-424, 2013

13. Guide for the Care and Use of Laboratory Animals. NIH Publication no. 85-23. Revised 1985.

14. Seldinger SI: Catheter replacement of the needle in percutaneous arteriography; A new technique. Acta Radiol 39: 368-376, 1953.

15. Dinc H, Kuzeyli K, Kosucu P, Sari A and Cekirge S: Retrieval of prolapsed coils during endovascular treatment of cerebral aneurysms. Neuroradiology 48: 269-272, 2006.

16. Vora N, Thomas A, Germanwala A, Jovin T and Horowitz M: Retrieval of a displaced detachable coil and intracranial stent with an L5 merci retriever during endovascular embolization of an intracranial aneurysm. J Neuroimaging 18: 81-84, 2008.
17. Kabbani MR, Smith A and Leider M: Endovascular coil retrieval using a trevoprovue stentriever. J Neurointerv Surg 7: e19, 2015.

18. Lee CY: Use of wire as a snare for endovascular retrieval of displaced or stretched coils: Rescue from a technical complication. Neuroradiology 53: 31-35, 2011.

19. Standard SC, Chavis TD, Wakhloo AK, Ahuja A, Guterman LR and Hopkins LN: Retrieval of a guglielmi detachable coil after unraveling and fracture: Case report and experimental results. Neurosurgery 35: 994-998, 1994.

20. Watanabe A, Hirano K, Mizukawa K, Kamada M, Okamura H, Suzuki Y and Ishii R: Retrieval of a migrated detachable coilcase report. Neurol Med Chir (Tokyo) 35: 247-250, 1995.

21. Koseoglu K, Parildar M, Oran I and Memis A: Retrieval of intravascular foreign bodies with goose neck snare. Eur J Radiol 49: 281-285, 2004.

22. Prestigiacomo CJ, Fidlow K and Pile-Spellman J: Retrieval of a fractured Guglielmi detachable coil with use of the goose neck snare 'twist' technique. J Vasc Interv Radiol 10: 1243-1247, 1999.

23. Ding D and Liu KC: Management strategies for intraprocedural coil migration during endovascular treatment of intracranial aneurysms. J Neurointerv Surg 6: 428-431, 2014.

24. Gurley JC, Booth DC, Hixson C and Smith MD: Removal of retained intracoronary percutaneous transluminal coronary angioplasty equipment by a percutaneous twin guidewire method. Cathet Cardiovasc Diagn 19: 251-256, 1990. 